\title{
Doped graphene quantum dots for intracellular multicolor imaging and cancer detection
}

Elizabeth Campbell ${ }^{a}$, Md. Tanvir Hasan ${ }^{a}$, Roberto Gonzalez Rodriguez ${ }^{b}$, Giridhar R. Akkaraju ${ }^{c}$, and Anton V. Naumov ${ }^{* a}$

a. Department of Physics and Astronomy, Texas Christian University, Fort Worth, TX 76129, USA

b. Department of Chemistry, Texas Christian University, Fort Worth, TX 76129, USA

c. Department of Biology, Texas Christian University, Fort Worth, TX 76129, USA
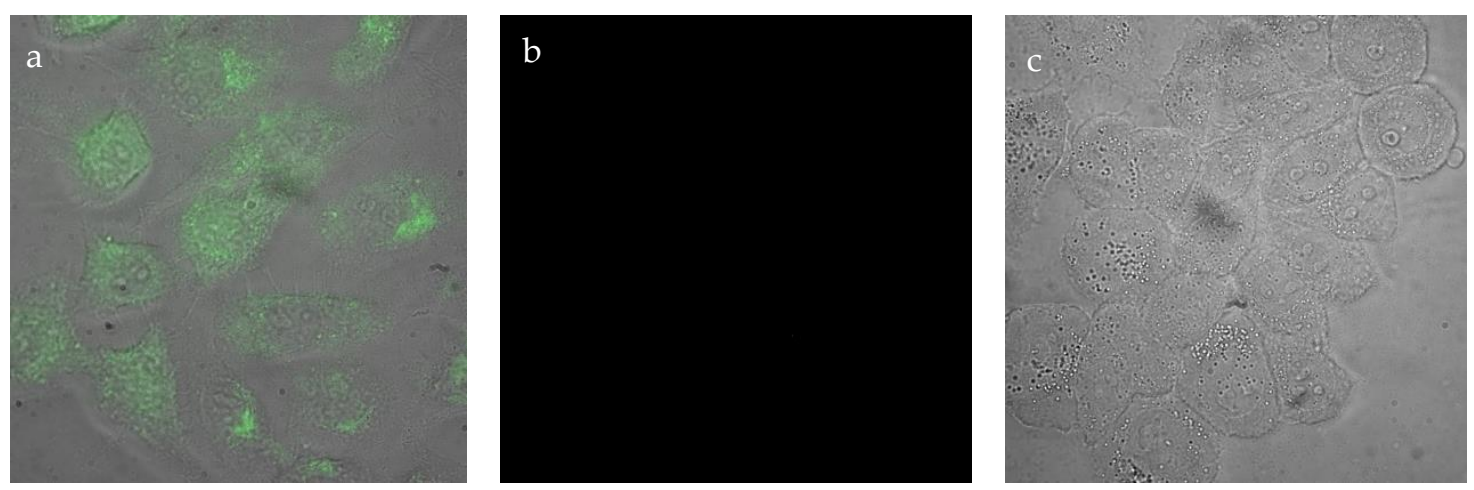

Figure S1: Fluorescence microscopy. (a) overlay of GQD emission with the bright field image of HeLa cells transfected with GQDs. $(b)(c)$ - Fluorescence and bright field images of non-treatment control HeLa cells. 


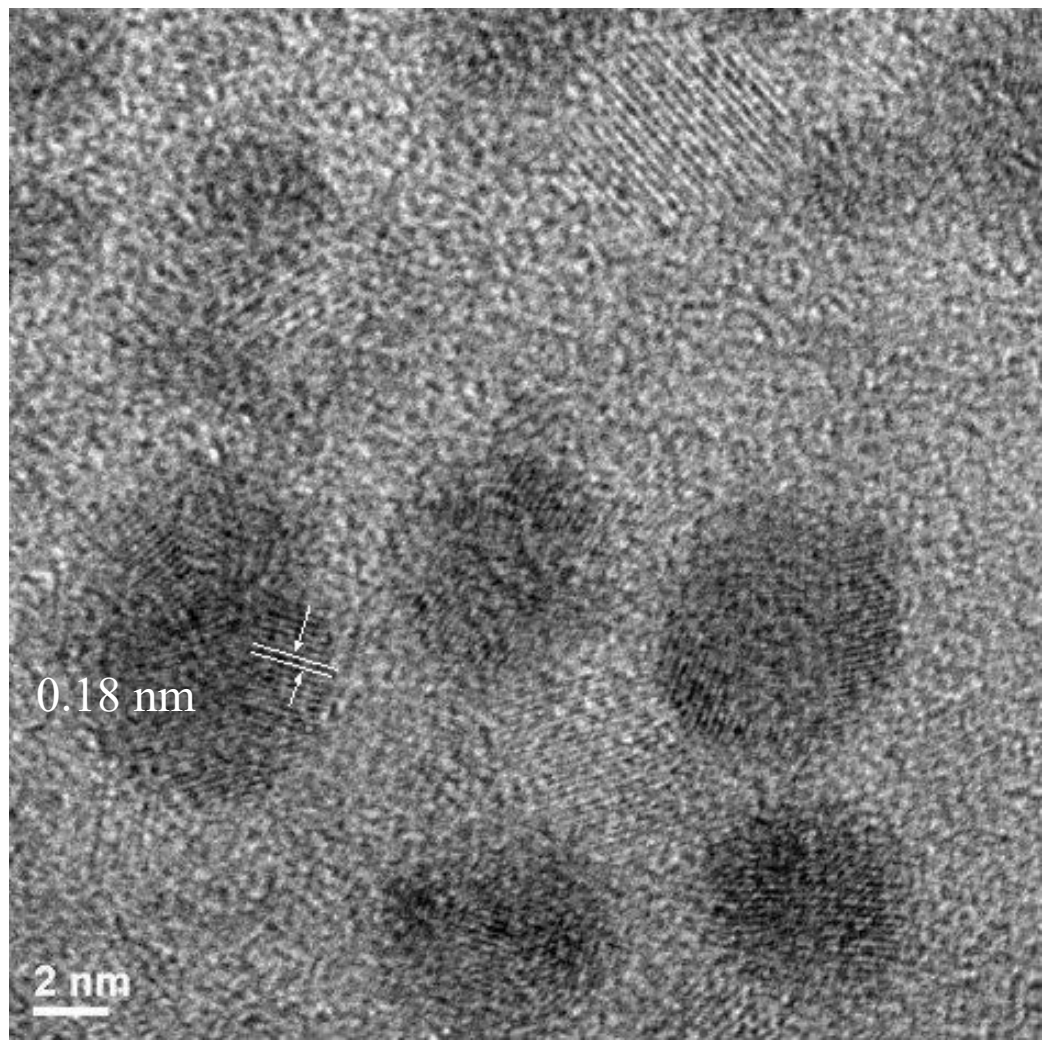

Figure S2: TEM image showing crystallinity of GQDs and discernable lattice domain structure.

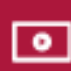

stack-3_MMStack_Pos0.ome_xvid.avi

Figure S3: Microscopy video of GQDs for 420 seconds, showing no photobleaching during that time. Imaged with $450 \mathrm{~nm}$ excitation and $535 \mathrm{~nm}$ emission. 


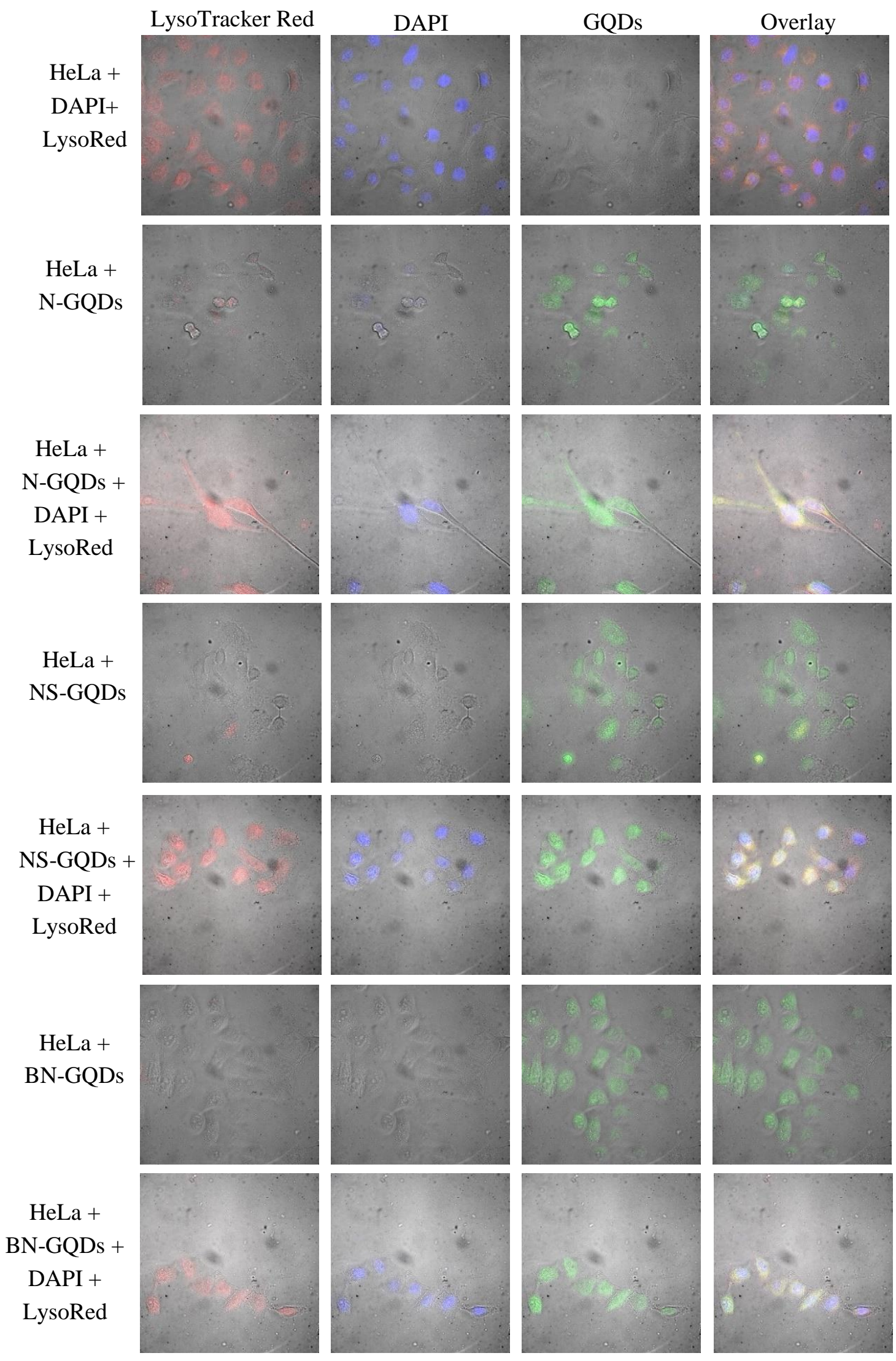

Figure S4: Colocalization image matrix showing GQD location within the cells for various GQD types and fluorescent staining. Red: LysoTracker Red, excitation at $540 \mathrm{~nm}$ emission recorded at $600 \mathrm{~nm}$; Blue: DAPI, excitation at $375 \mathrm{~nm}$ emission recorded at $450 \mathrm{~nm}$; Green: GQDs, excitation at $475 \mathrm{~nm}$ emission recorded at $535 \mathrm{~nm}$. 


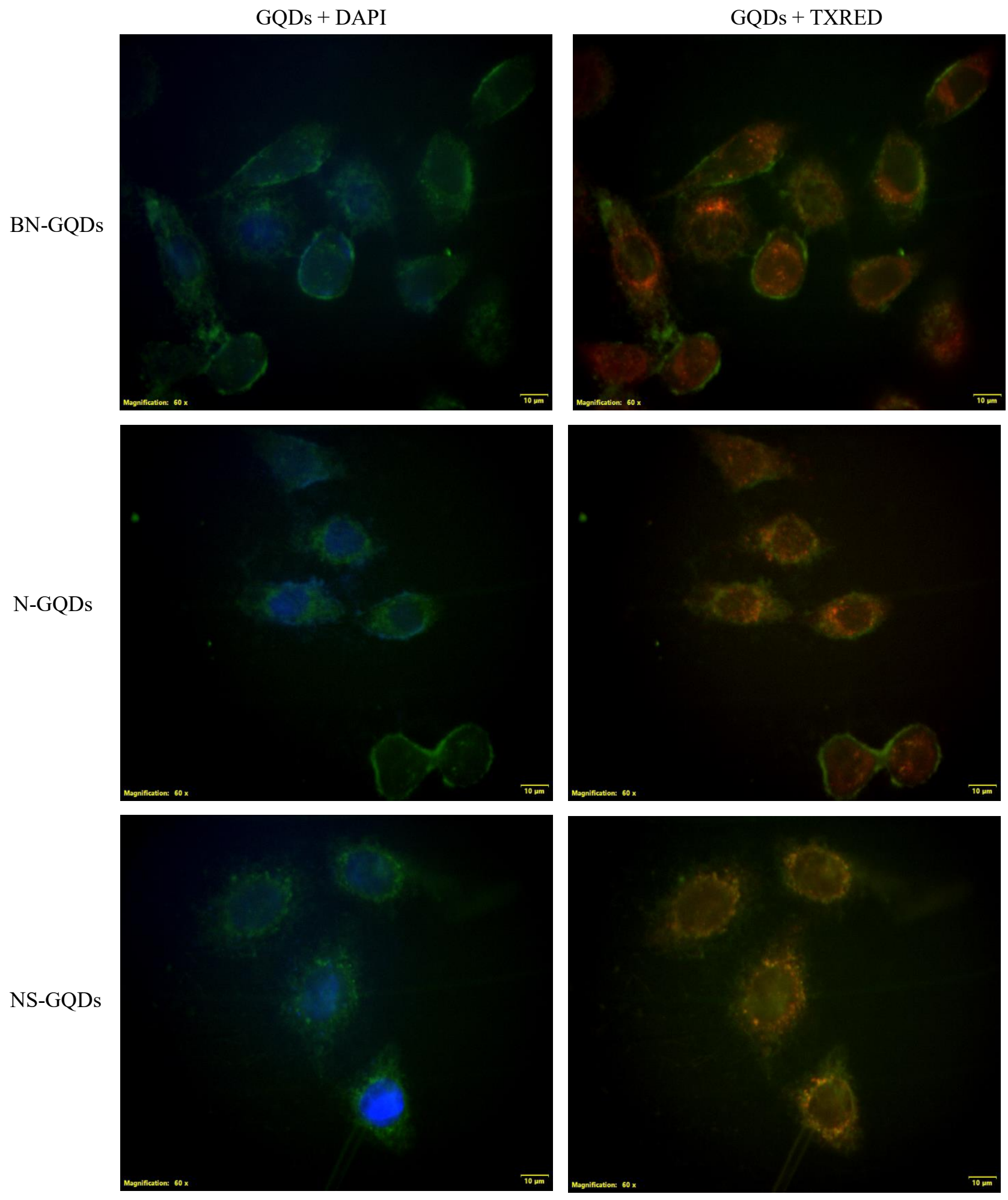

Figure S5: Confocal overlay image matrix indicating localization in the lysosomes and some in the nucleus; Texas Red exc. 559 nm em. 630 nm; DAPI exc. 375 nm em. 450 nm; Green Fluorescent Protein exc. 469 nm em. 525 nm. 


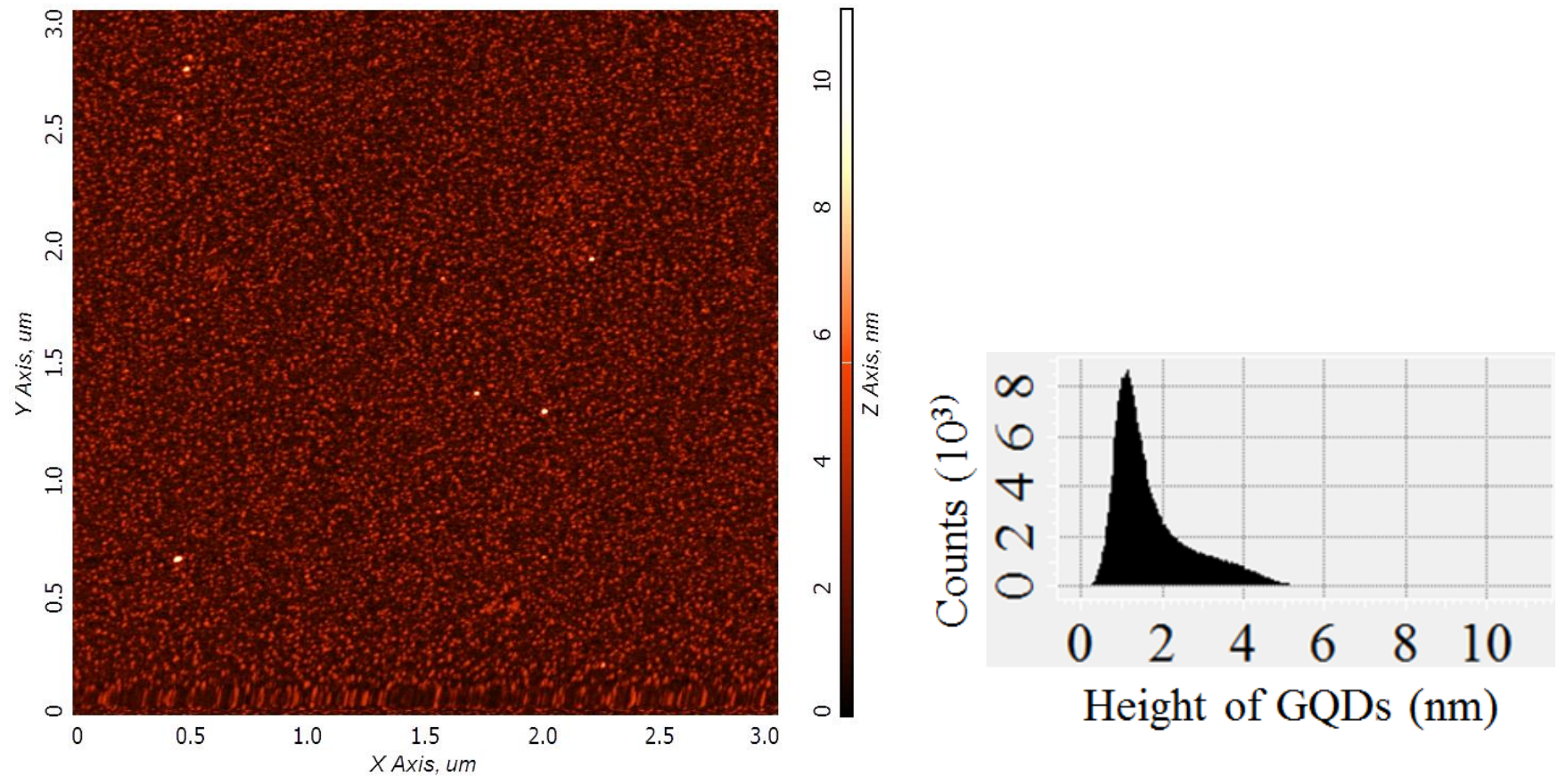

Figure S6: AFM of nitrogen-doped graphene quantum dots. Mean height=1.82 nm. 
Blue

pH 6.0

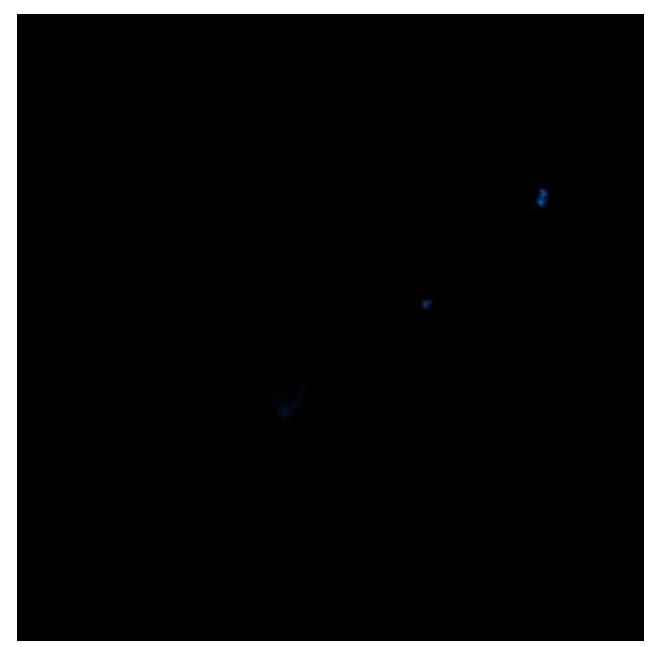

$\mathrm{pH} 8.5$

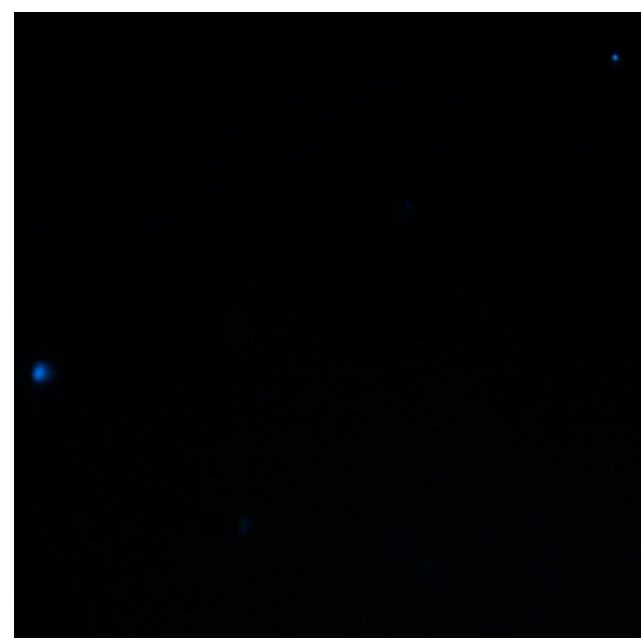

Green
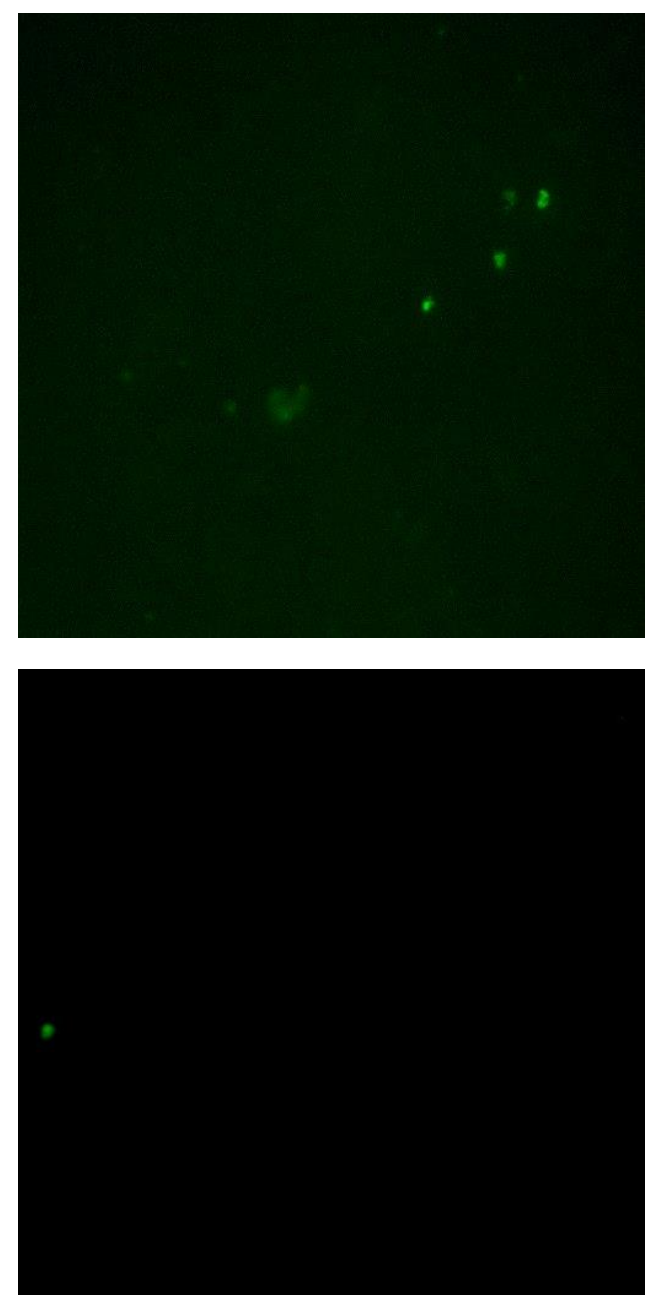

Figure S7: Images of N-GQDs at varying pH levels. Blue imaged with excitation $375 \mathrm{~nm}$, emission at $450 \mathrm{~nm}$. Green imaged with excitation $475 \mathrm{~nm}$, emission at $535 \mathrm{~nm}$.
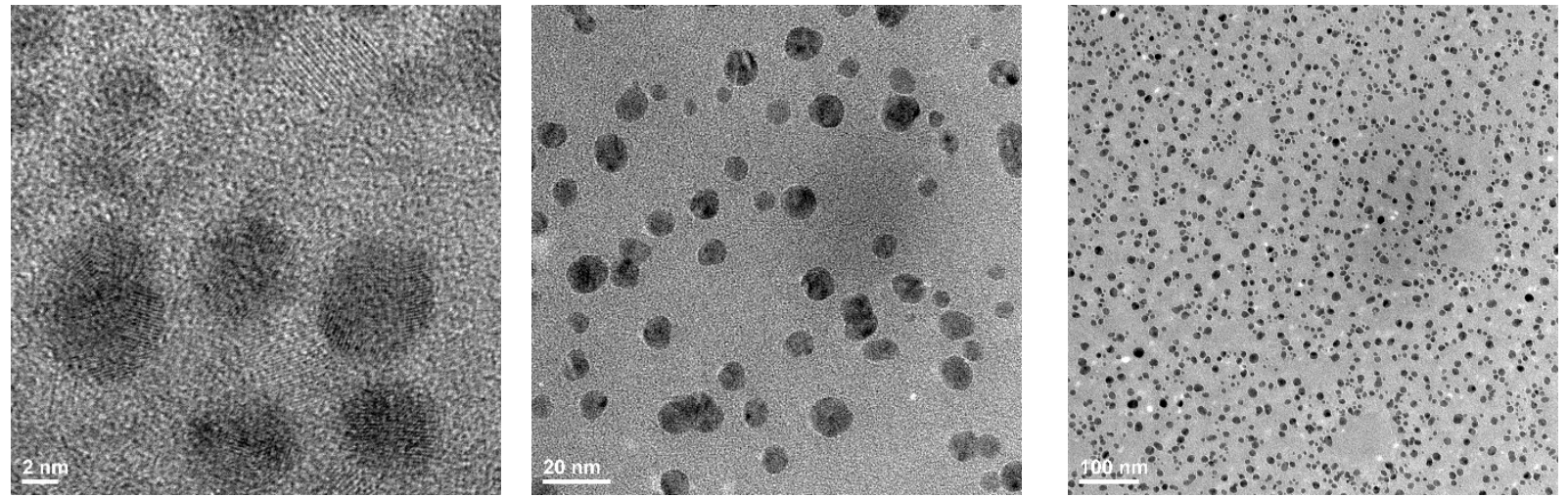

Figure S8: TEM images of N-GQDs at varying magnifications. 

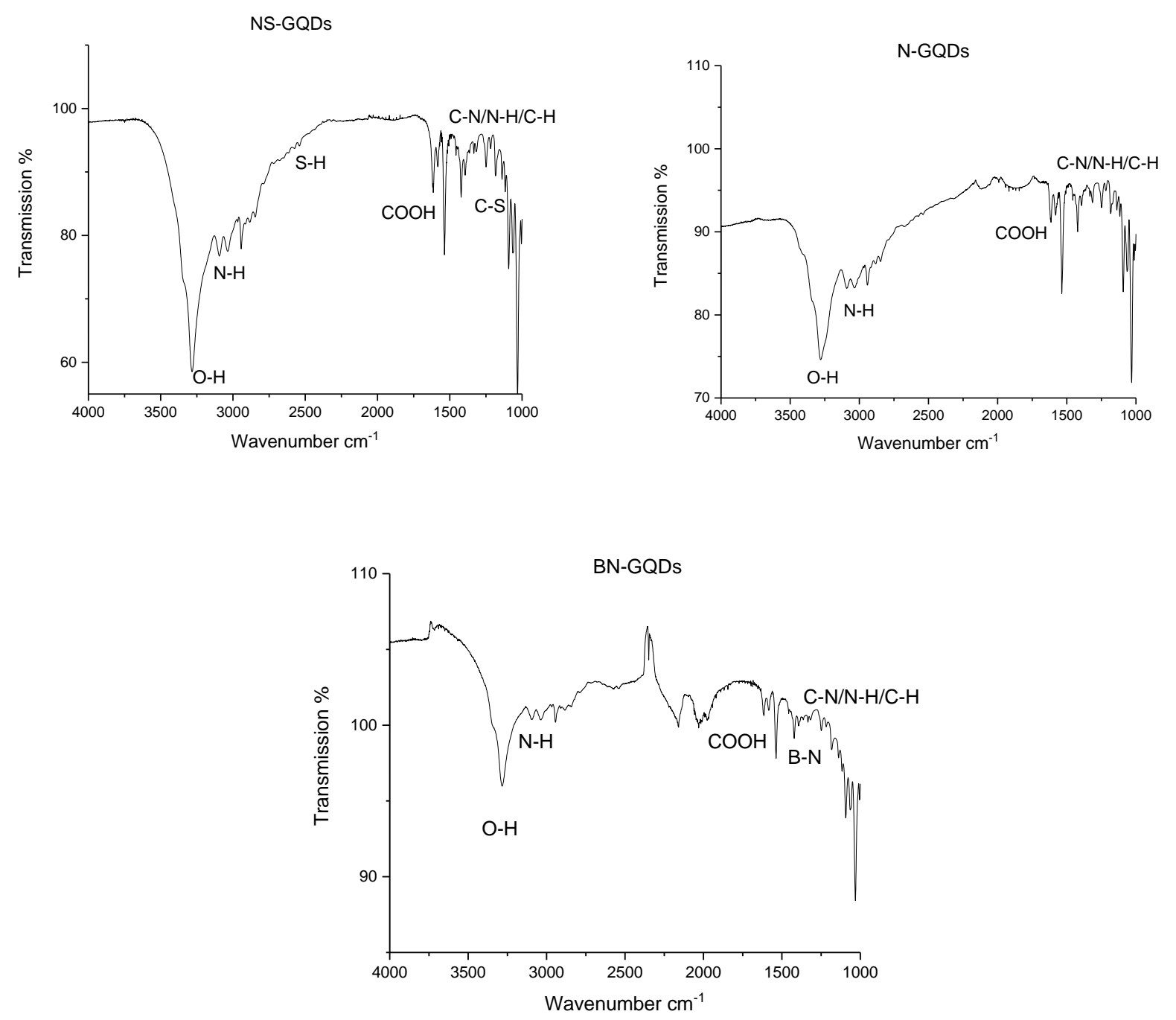

Figure S9: FTIR spectra of N-GQDs, NS-GQDs, and BN-GQDs. 
A

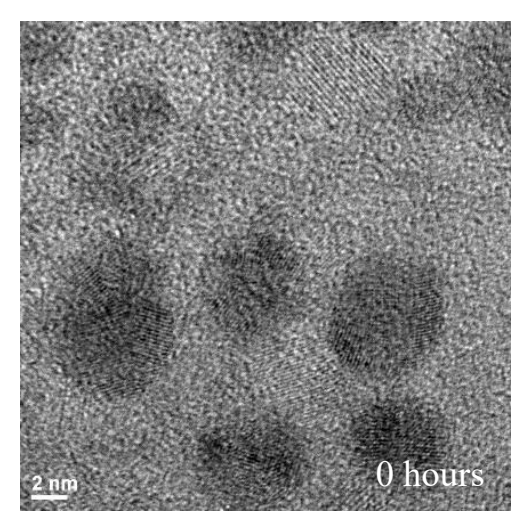

$\mathrm{C}$

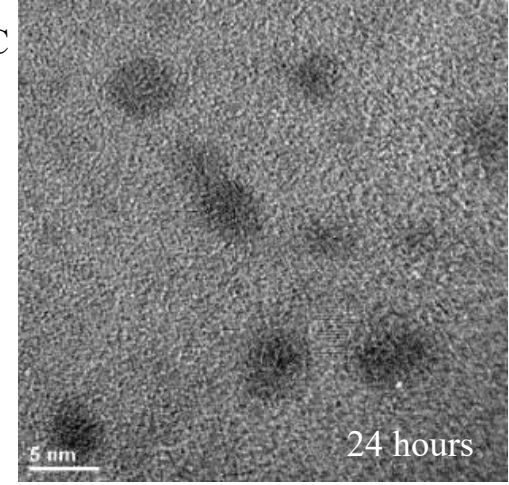

$\mathrm{E}$

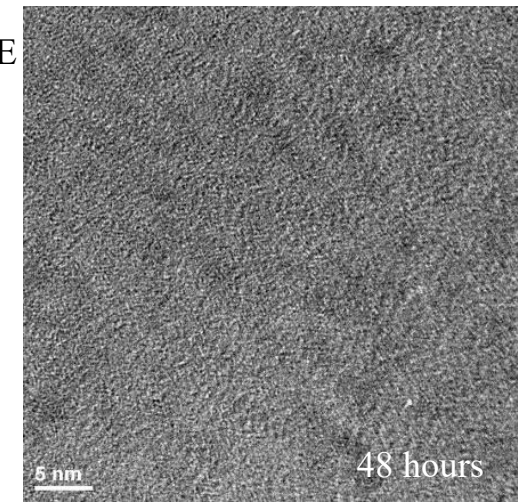

B
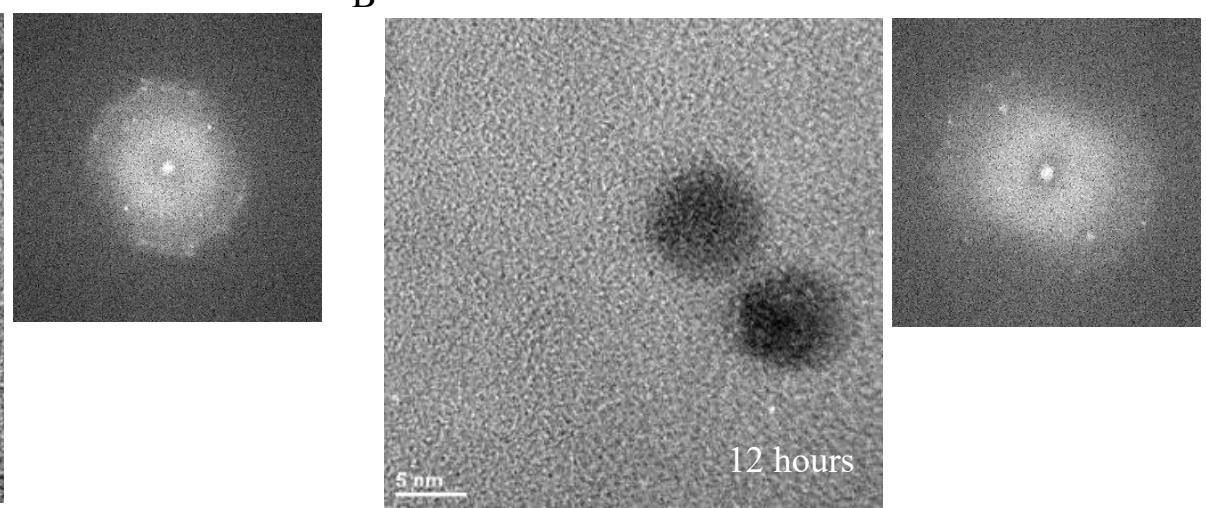

$\mathrm{D}$
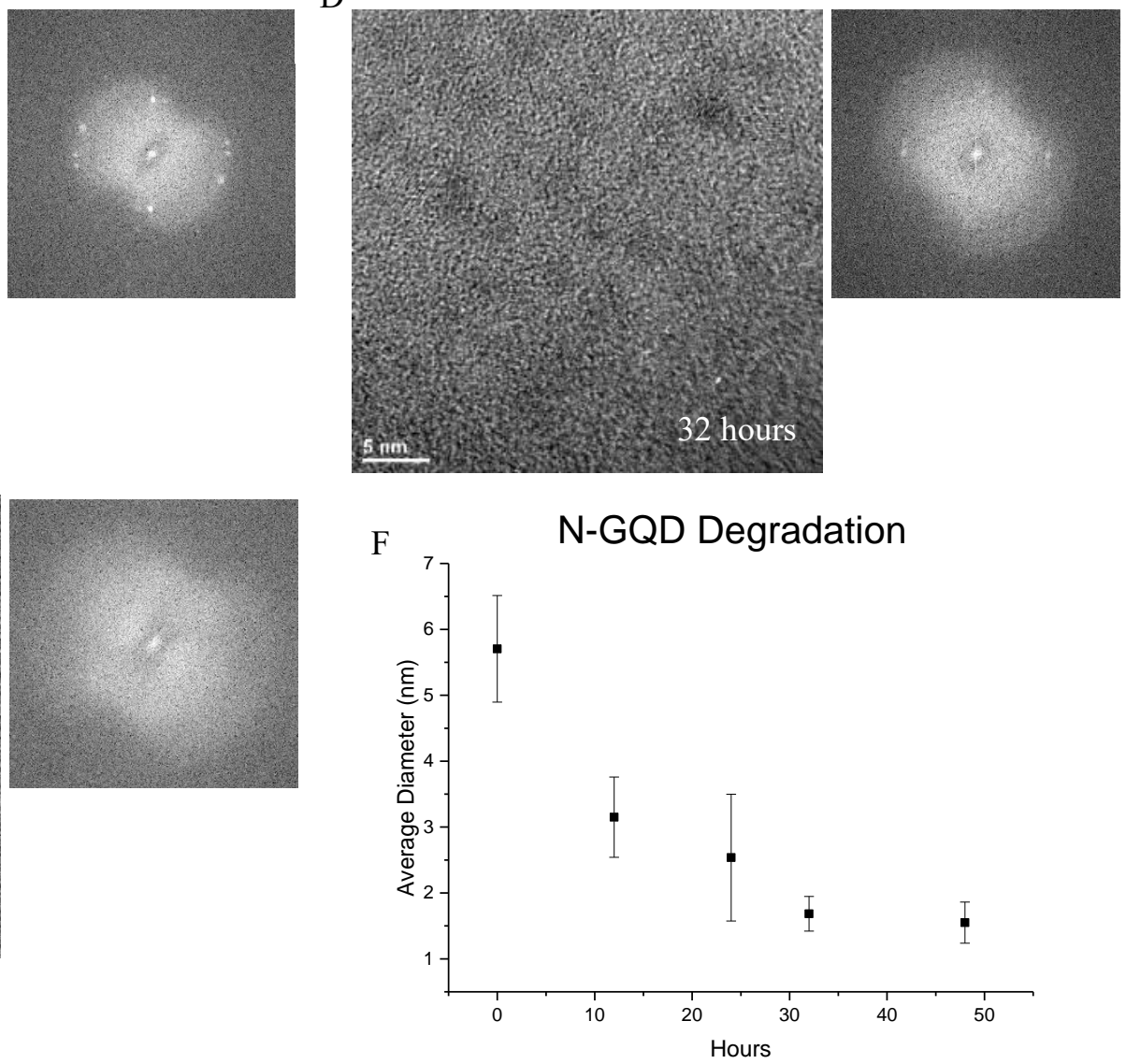

Figure S10: (A-E)-TEM images of N-GQDs in HeLa cell culture at varying times with the corresponding FFT image analysis, indicating the crystallinity of the structure exposed to cell media at different times; $F$-Average diameter of $N$ GQDs with respect to time, analyzed using TEM images at varying times for over $100 \mathrm{~N}$-GQDs in HeLa cell culture, indicating degradation of quantum dots. 